\title{
APROXIMACIÓN CONCEPTUAL DE LA SEGREGACIÓN SOCIO ESPACIAL Y RESIDENCIAL EN CIUDADES INTERMEDIAS EN AMÉRICA LATINA
} CONCEPTUAL APPROXIMATION OF THE SPACE AND RESIDENTIAL PARTNER SEGREGATION IN INTERMEDIATE CITIES IN LATIN AMERICA

\author{
Wilmer Yesid Farfán Tocarruncho ${ }^{1}$ \\ Universidad de Ciencias Aplicadas y \\ Ambientales, UDCA, Colombia
}

\section{RESUMEN}

El objetivo de este artículo es precisar que la segregación socio-espacial y residencial en las ciudades intermedias contemporáneas en América Latina se diferencia en la conceptualización y aplicabilidad en el espacio. Para ello, se realizó una revisión documental

$1 \quad$ Estudiante de Maestría en Geografía UPTC IGAC. Licenciado en Ciencias Sociales de la Universidad Pedagógica y Tecnológica de Colombia; Administrador Público de la Escuela Superior de Administración Pública; Especialización en Proyectos de Desarrollo -Escuela Superior de Administración Pública; Profesor de la Universidad de Ciencias Aplicadas y Ambientales UDCA. Monitor Escolar - Banco Mundial. Colombia. Integrante Semillero de Investigación GEOT. Wilmerfarfan86@hotmail.com ORCID: https://orcid.org/0000-0002-3444-7236 que incluyó artículos, tesis e informes de instituciones afines a la temática publicados entre 1900 y 2018 que definían o utilizaban como categorías de análisis la segregación socio-espacial y residencial. Se encontró, en primer lugar, que los procesos de segregación socio-espacial y residencial están articulados con el sistema económico, la globalización y las prácticas neoliberales que existen en el territorio y el espacio; en segundo lugar, la población se ubica/aglomera según sus condiciones socioeconómicas permitiendo que las personas se asienten en determinados lugares, caracterizados como homogéneos; en tercer lugar, se diferencia el potencial de 
la renta lo cual antepone una desvalorización, desprestigio o deterioro del territorio, o por el contrario una valoración de algunos sectores permitiendo así la fragmentación territorial. Se podría concluir que la ubicación de los sitios que son segregados se ubica particularmente en la periferia de las ciudades y un efecto dado a esto se dan os problemas de inseguridad que en algunos aspectos perjudica la calidad de vida.

\section{PALABRAS CLAVE:}

segregación socio espacial, residencial, espacio, territorio, aglomeración.

\section{ABSTRACT}

This article objective is to identify that socio-spatial and residential segregation in contemporary intermediate cities in Latin America differs in conceptualization and applicability in space. For this purpose, a documentary review was carried out that included articles, theses and reports from institutions related to the subject published between 1900 and 2018 that defined or used as categories of analysis the socio-spatial and residential segregation. It was found that: first, socio-spatial and residential segregation processes are articulated with the economic globalization and

neoliberal system, practices that exist in the territory and space; secondly, that population is located / agglomerates according to their socioeconomic conditions allowing people to settle in certain places, characterized as homogeneous; thirdly, the income potential puts a devaluation, discredit or deterioration of the territory, or on the contrary a valuation of some sectors. It is right to conclude that site's location that are segregated is particularly located on the periphery of the cities and an effect given to this presents problems of insecurity.

\section{KEYWORDS:}

socio-spatial, residential, space, territory, agglomeration segregation

\section{INTRODUCCIÓN}

En este artículo se identifica las principales características que permiten conceptualizar que la segregación socio-espacial y residencial en las ciudades intermedias contemporáneas en América Latina, se modifica según su campo de acción y metodología, permitiendo establecer que estos dos fenómenos urbanísticos son completamente diferentes y que de ahí se establecen criterios propios a desarrollar en el terreno.

El escenario actual de América Latina exige la implementación y articulación de nuevas estrategias conceptuales que permitan alcanzar un desarrollo humano sostenible. Una de estas, es la de poder consolidar un ordenamiento territorial, dado a las realidades y necesidades que se presentan continuamente en el espacio, permitiendo implementar acciones tendientes a revertir impactos negativos en el territorio, bajo directrices de la buena planificación. Para ello se podría iniciar identificando los conceptos que se están llevando a cabo para estudiar aquellos fenómenos urbanísticos, con el fin de analizar las realidades del entorno y así mismo entrelazar las necesidades con las posibles soluciones, alcanzando la efectividad en la planificación de excelentes políticas que beneficie el orden del espacio.

La anterior afirmación se fundamenta en hechos en el desconocimiento y confusión conceptual de la segregación espacial, socio-espacial y segregación residencial. De esta forma partimos de identificar que la segregación es un fenómeno urbanístico que se podría estar presentando como consecuencia de los procesos neoliberales que se desarrollan a nivel mundial y del sistema 
económico que predomina en América Latina. "El concepto de segregación ha sido tornado de una forma como absoluto, la cual no requiere de investigación empírica ni explicación científica en las ciudades latinoamericanas, asociándolas en el mayor de los casos a la marginalidad ejercida de un estrato de la población de mayores recursos socioeconómicos sobre una de menores ingresos y asociadas a la informalidad". (Roca Miguel 2012, p 121). De igual manera suele asociarse como sinónimo de separación, aceptado que su aplicabilidad tiende a cambiar.

Partiendo de lo anterior el escrito se encamina en identificar de manera conceptual la realidad que se maneja en este momento sobre la segregación espacial, socio-espacial y residencial para el caso de América Latina.

\section{FUNDAMENTOS TEÓRICOS DE LA SEGREGACIÓN}

A partir de las últimas décadas del siglo $X X$, la preocupación central en identificar que fenómenos urbanísticos se presentan en el territorio, ha redefinido los conceptos de segregación social, socio-espacial y residencial, ha dado objeto a una renovación conceptual y metodológica sin precedentes.

El cambio sustancial esta dado al concepto de segregación sin apellido a un fenómeno netamente urbano, sin embargo con el tiempo se tomó nuevas tipologías que permitieron añadirles la parte social, como un fenómeno netamente humano. Al comprender las relaciones de la segregación socio-espacial, residencial y su desarrollo en el espacio, establece un punto de inicio, que permitirá aproximar los fenómenos a los conceptos. Debido a que algunos autores confunden los significados de la segregación socio-espacial y la segregación residencial, dándolos como iguales. Al respecto, la definición conmensurablemente aceptada de segregación espacial es: «el grado en el que dos o más grupos sociales viven de manera separada el uno del otro, en partes distintas del espacio urbano» (Massey \& Denton, 1988, p. 282). Por su parte existen diferentes acepciones sobre la segregación socio-espacial, que a mi modo ver son aceptables, frente al fenómeno que está creciendo en América Latina. Una de las definiciones más aceptadas afirma que es "la existencia de una diferenciación o distribución desigual de ciertos grupos sociales al interior del espacio urbano" (Brun, 1994, p. 22). Esta definición dada por el autor, da luces para poder analizar del por qué de este artículo, señalando así, que la segregación socio-espacial en términos de la segregación que verifica por las condiciones de localización del lugar de residencia y también por razones de ingresos económicos que dificultan el acceso para los más pobres no solamente a la vivienda (Rubiano y Burbano, 2015), sino también a su entorno y sus implicaciones en relación con la movilidad cotidiana de los individuos en el espacio. Con base al anterior análisis se puede mencionar que la segregación socio-espacial es un fenómeno urbano, cuyo procesos están asociados de manera gradual a las condiciones económicas, acceso a lugares de ocio, beneficiando o desplazando a ciertos grupos y con ellos a sectores que no tienen ciertas condiciones que permiten su adquisición.

Desde otro punto de vista, Arriagada y Rodríguez (2003, p. 11) sostienen que el conflicto de la segregación espacial se analiza desde las concepciones racionalesquetomanlosindividuos de los grupos sociales para actuar como sujetos económicos, es decir, actúan por la búsqueda de maximizar su utilidad, la exclusividad, la distinción, la afinidad, la acumulación de activos, la construcción de redes o el acceso a recursos, es decir analizan el espacio como un momento de exclusividad bajo las directrices del valor y uso del suelo. Como consecuencia de esto, es común observar que según los ingresos o 
el nivel económico de la sociedad, se permita escoger en que parte del territorio se puede asentar y los que no tienen las posibilidades de escoger, se tienen que someter a ciertos criterios de dominación que están dados a sus ingresos. La afirmación se sustenta en el desarrollo de grandes capitales inmobiliarios permitiendo el surgimiento y apogeo de un nuevo proceso de desarrollo habitacional: como se mencionó anteriormente, el barrio cerrado (Sabatini, 1997; Sabatini \& Cáceres, 2004; Hidalgo, 2004; Sabatini \& Salcedo, 2007; Sabatini, Wormald et al', 2007). Este proceso habitacional esta incursionado por las nuevas formas de vivienda con privilegios a los estratos medio y alto, dando procesos de desfragmentación territorial en la periferia de la ciudad. Cuyas situaciones permiten generara situaciones de aislamiento, rechazo, incertidumbre en aquellas poblaciones de ingresos bajos. En cuanto a la segregación residencial es detallada y conceptualizada de diferentes formas (Bayona i Carrarsco 2007). Por ejemplo, para Aguilar y Mateos $(2011,7)$ la segregación residencial es "la aglomeración o distribución de los grupos sociales de una misma condición socioeconómica en el espacio", mientras que para Castells (1986, 204) es "la tendencia a la organización del espacio en zonas con fuerte homogeneidad social interna e intensas disparidades entre ellas, dándose esta disparidad no solamente en términos de diferenciación sino también en términos jerárquicos". De la revisión de estas y otras definiciones se observa que el concepto que nos ocupa suele utilizarse indistintamente para describir lo mismo situaciones estáticas que dinámicas, también suele pasar de ser un término descriptivo a ser uno explicativo, y de actuar como indicador a ser causa de diversos aspectos de la injusticia social (Bayona i Carrarsco 2007, Brun 1994).

La ciudad latinoamericana podría presentar una de las características de segregación, dado a la presencia de espacios homogéneos, determinada por las condiciones en que están rodeados. Entendida de esta forma, la segregación residencial ocurre cuando población de un mismo color, clase social, religión, o cualquier otro grupo específico muestra una tendencia a vivir de manera próxima en un área de la ciudad, caracterizado por tener las mismas características en los hogares donde se están ubicadas estas viviendas.

Si diferenciamos la segregación residencial con la espacial, se podría mencionar que la residencial está bajo unas premisas del espacio, con ciertas discrepancias que están relacionadas de manera particular, cosa contraria pasa con el segregación espacial, donde las diferencias sociales se pueden ver plasmadas en una división del espacio. Es así que en las ciudades antiguas el espacio urbano se dividió según el estatus social y la adscripción a distintos clanes (Sjoberg 1960). Desde aspectos históricos algunos grupos se han ubicado y agrupado en espacios jerarquizados, con niveles de poder en ciertas particularidades plasmadas en un dominio político, religioso, militar y económico de la sociedad. En algunos casos extremos, algunos grupos específicos fueron separados y estigmatizados como no deseables, y en consecuencia, confinados a áreas específicas de la ciudad.

Desde la década de 1980 los estudios sobre segregación residencial han cobrado importancia central para comprender la marginalidad urbana y su reproducción; y es que la variable espacio acaba teniendo un peso central en el continuo deterioro de los barrios marginales. En el gueto negro norteamericano, donde el fenómeno es más drástico, es el efecto concentración (concentration effect), ser una población que vive junta. En espacios extremadamente segregados y en las peores condiciones de vida, acaba llevando a este grupo a desarrollar una forma de vida alternativa y paralela a la del resto de la sociedad (Wilson 1987: 58). Del mismo 
modo, en un estudio comparativo de más de cincuenta ciudades norteamericanas, Massey y Denton (1993: 9) afirman que la segregación espacial es el principal factor estructural responsable de la perpetuación de la pobreza de la población negra en los Estados Unidos. Asimismo, la segregación del gueto negro crea para sus habitantes una situación en la que sus probabilidades de éxito disminuyen y más bien quedan atrapados en un círculo de pobreza y reproducción de su condición de clase (Massey y Denton 1993: 181)

\section{CONCEPTUALIZACIÓN DE LA SEGREGACIÓN SOCIO-ESPACIAL, ESPACIAL Y RESIDENCIAL EN AMÉRICA LATINA}

Para el caso de Colombia, la segregación socio-espacial es entendida como el proceso social donde una clase o cualquier otro grupo específico manifiesta la tendencia de vivir de manera próxima en un área de la ciudad en común, relacionada a los aspectos económicos y de ocio, permitiendo así la creación de espacios comunes, aglomerados y homogéneos, que en ocasiones se convierten en zonas cerradas y dominadas por un grupo. En el caso de Lima, al igual que en la mayoría de las ciudades latinoamericanas la segregación se acentúa aún más por las diferencias socioeconómicas por encima de las diferencias raciales (rasgo característico de la segregación en los ghettos de Norteamérica) (Roca Miguel 2012, p 121). Es así, como se puede constatar espacial y socialmente: dado a que el primer caso, los grupos humanos se distribuyen desigualmenteen el espacio con respecto a otros; y en el segundo, cuando estos no se integran socialmente con los demás (Rodríguez, 2001:11). Estas dos últimas tipologías del fenómeno pueden entenderse o no en el espacio geográfico. Un caso particular esta dado en la sociedad India, en donde los grupos humanos pertenecientes a distintas castas cohabitan sin mezclarse socialmente, y $\sin$ que la distancia espacial implique la desaparición de la distancia social, lo cual es conocido como segregación por exclusión (Sabatini, 1993:3). De esta manera se da otro tipo de tipo de segregación, bajo parámetros raciales o por exclusión, como lo ocurrió en Sudáfrica durante el Apartheid, cuyo fenómeno está dado a la segregación forzada (Sabatini, 1999:3). Y de igual forma las adquiridas por el fenómeno, es la segregación por localización de grupo, la cual se presenta cuando un grupo social muestra un patrón de distribución global, a saber, cuando sus miembros se localizan en un área determinada del territorio, en presencia o no de otros grupos sociales (Sabatini, 1999:3).

Dado lo anterior se manifiesta que las tipologías de la segregación por exclusión, forzada y por localización de grupos, está dada a excluir cualquier grupo social, que se establezca en el espacio, permitiendo así la existencia de las desigualdades, inequidades de unos sobre otros, por el simple de hecho de pertenecer a un grupo establecido o jerarquizado.

Por otro lado la definición conmensurablemente aceptada de segregación espacial es: «el grado en el que dos o más grupos sociales viven de manera separada el uno del otro, en partes distintas del espacio urbano» (Massey \& Denton, 1988, p. 282). Lo anteriormente mencionado por Massey \& Denton viene siendo cuestionado con sus diferentes y contradictorias acepciones, y aparece en la actualidad como un fenómeno natural, dado a las condiciones socio-económicas que presentan las familias los grupos, que en ocasiones presenta de alguna forma tendencias y transformaciones de las ciudades capitales e intermedias, y en articular en Colombia.

Se debe pensar que el concepto de segregación es un instrumento teórico y no técnico, a 
diferencia de la marginalidad ${ }^{2}$, conceptos que generalmente se asocian para poder explicar las dinámicas de la sociedad en un espacio. La marginalidad se caracteriza por ser ambiguo y polisémico (Cingolani, 2009).

En este marco, y para Germani (1980),

La marginalidad constituye una de las perspectivas desde las cuales puede abordarse la problemática de la modernización, de los aspectos sociales y humanos del desarrollo $y$ de la problemática generada por los contrastantes modelos de procesos sociales y sociedades que se proponen como respuesta o solución a los problemas del mundo contemporáneo, tanto en sus áreas centrales como, $y$ sobre todo, en las periféricas ( $p$. 34).

Desde la perspectiva del autor, la situación de marginalidad supone la existencia de cierta forma de pertenencia y de relación del grupo marginal con respecto a la sociedad de la cual el sector es excluido por otro grupo marginal. En este sentido, la marginalidad tendría un carácter relativo y de manera constante, dependiendo del modelo de participación y de grados o intensidad. Para Manuel Castells (1998, p. 203) define la segregación urbana como: «[...] la tendencia a la organización del espacio en zonas de fuerte homogeneidad social interna y de fuerte disparidad social entre ellas, entendiéndose esta disparidad no solo en términos de diferencia, sino de jerarquía». En ese sentido Castell manifiesta que la segregación tiende a formar grupos jerárquicos que van a dominar el espacio y las dinámicas de ellas, permitiendo así el surgimiento de jerarquías establecidas por

$2 \quad$ Un antecedente al concepto de marginalidad acuñado en América Latina puede encontrarse en la noción de marginal man, nacida en el interior de la Escuela de Chicago en las décadas del 20 y 30. Dentro de esta tradición, iniciada por Robert Park y Everet Stonequist, el marginal era aquel individuo que se encontraba al margen de dos culturas y no se identificaba plenamente con ninguna de ellas. grupos dominantes, generando de alguna forma caos e inseguridad dentro de ellas mismas.

Mientras que Francisco Sabatini (2003) sintetiza a la segregación como la «aglomeración en el espacio de familias de una misma condición social». Haciendo a la vez referencia a tres dimensiones inmersas en el concepto de segregación:

1. El grado de concentración espacial de los grupos sociales. Es un recurso usual para consolidar nuevas identidades o defender las viejas identidades amenazadas por la presencia de nuevos enclaves. Impacto positivo: fomenta las identidades culturales.

2. Grado de homogeneidad social de un área (del área en que el grupo se concentra).La sociedad no existe fuera del espacio. El espacio adquiere entonces una significación social y no un mero escenario o soporte físico de los acontecimientos.

3. Segregación subjetiva (percepción de la segregación por parte del ciudadano). Determinada por los aparatos ideológicos, tales como los medios de comunicación, prensa escrita o la percepción de los ciudadanos sobre determinado espacio.

Por lo tanto la segregación solita, sin apellidos, debe ser entendida como un hecho histórico y a la vez espacial, cuyo concepto deriva de factores ideológicos generalmente aceptados por sus afectados. Tendiente a tener una nueva dinámica, producto de los procesos que lo influencian, como la globalización y la transformación económica.

Esta visión de segregación requiere operar cambios estructurales para dar cuenta de aspectos diferenciables que tienen distintos 
intereses, tanto en términos de sus impactos sociales, económicos y urbanos con incidencia desde la política pública. Donde las dos primeras dimensiones están dadas a ser objetivas de la segregación. Donde el proceso está proporcionado a registrar de forma simple el fenómeno y no se especifica de forma sustancial, dado a que se pueden presentar índices generales en distintos grados en importancia numérica de cada grupo social, en un conjunto de la ciudad, sea las variaciones de los barrios y áreas internas.

La tercera dimensión, es relativa al grado de prestigio de los barrios, dado a que es de carácter subjetivo. Hace referencia a las imágenes, reputación, percepciones y estigmas territoriales asignados por la población de la ciudad a algunos de sus vecindarios. Como es el caso del prestigio que se ocasiona en algunos barrios como consecuencia de los negocios inmobiliarios, donde se establece una estrecha conexión entre la capitalización del suelo, por medio de la renta. $Y$ en contraparte están los barrios que se le atribuyen formas de vivienda donde la desintegración de los espacios es estigmatizada.

Para Samuel Jaramillo en su obra "Hacia una teoría de renta del suelo urbano (2008)"

La configuración física irracional de las aglomeraciones urbanas, el encarecimiento de la vivienda y del espacio construido en general, la expulsión de población de sus asentamientos tradicionales, la resistencia de los grupos implicados en estos desplazamientos, la especulación, la destrucción inclemente de secciones enteras de las ciudades que son la base de la memoria colectiva, la hiperdensificacion, la congestión, etcétera, todos ellos son problemas urbanos que han llegado a convertirse en signos de nuestro tiempo.(Jaramillo Samuel, 2008, p XVII)

Actualmente el estudio de la segregación reconoce fundamentalmente varias líneas de análisis dadas por Johnston, Massey y Denton, Sabatini y Sierralta. Premisas que parten de la tendencia de un grupo a concentrarse en algunas áreas; la conformación de áreas socialmente homogéneas; la percepción subjetiva que tiene la gente de las dimensiones objetivas de la segregación, como producto de los procesos del urbanismo.

El término segregación socio-espacial tiene una fuerte carga polisémica, que ha permitido una extensa comprensión literaria, haciendo énfasis a la existencia de diferencias 0 desigualdades sociales dentro de un colectivo urbano. Actualmente el fenómeno puede interpretarse desde los cambios políticos, económicos, sociales, culturales prolongado en las diferentes ciudades, ocasionados desde hace unas décadas, la conservación de los rasgos propios a la ciudad actual. Al contrario, se trata de una noción compleja relacionado con múltiples aspectos sociales, económicos, culturales y del paisaje de las ciudades (Machado, 2001; Gaschet y Le Gallo, 2005).

Por otro lado la segregación residencial es un concepto que está ligado al fenómeno de aglomeración en el espacio de familias de una misma condición social, más allá de cómo definamos las diferencias sociales. La segregación puede ser atribuida según las condiciones étnicas, origen migratorio, etaria o socioeconómica, que en esta última estimación, prevalece en ciudades de América Latina.

De allí que se puede afirmar que en palabras de Lévy y Brun, este concepto 
remite a "las formas de desigual distribución de grupos de población en el territorio" (2002, p. 147). De ahí, que pueda ser pensada como una de las formas en que se expresa el proceso de diferenciación social o, lo que es igual, como la cristalización en el espacio de la estructura social (Machado Barbosa, 2001).

Para Harvey (1992), las ciudades en la actualidad han dejado de planificarse en su conjunto para sólo abocarse a diseñar partes de él, como resultado de la especulación inmobiliaria y sin ningún tipo de previsión; advierte que aparecen fenómenos ajenos al problema y que no se planifican desde el escritorio, sino por el contrario, se dan desde las mismas entrañas del fenómeno, como la miseria, pobreza y la corrupción; y por el otro, se dan nuevas edificaciones que tratan de mitigar este fenómeno, dado a la construcción de conjuntos cerrados. Que Harvey ha formado una especie de islas o conjuntos cerrados exclusivos que sin lugar a dudas, dificultan la integración urbana y aumentan el aislamiento social en medida que los ricos se hacen más ricos y los pobres más pobres. Desde sus inicios la segregación socio-espacial se ha relacionado íntimamente con otros fenómenos como la segregación residencial, segregación territorial, desigualdad, inequidad, pobreza, polarización, vulnerabilidad $\mathrm{e}$ inseguridad. Aun estos momentos se continúa con esos adjetivos, pero actualmente el concepto de segregación socio-espacial implica sucesos y fenómenos más complicados, que están asociados a las dinámicas políticas neoliberales y de ahí su uso, a la hora de ser estudiado como fenómeno social. Los primeros estudios enfocados en el tema de la segregación residencial, fue utilizado como concepto para poder observar el proceso de concentración y "guetificación" de las personas de color en las ciudades norteamericanas, al igual que las zonas de asentamiento de los inmigrantes en dichas ciudades, se inicia con mayor fuerza a partir de la Sociología Urbana de la Escuela de Chicago, en las primeras décadas del siglo $X X$, a partir de los trabajos de Park, Burgees y Wirth, entre otros autores (Checa \& Arjona, 2007). La segregación residencial, por tanto, "implica que los diferentes grupos socioeconómicos de una ciudad o metrópoli viven de manera separada, con escasa o nula convivencia entre sí" (CEPAL, 2010, pág. 143)

En palabras de Lévy y Brun, el concepto de segregación residencial se remite a "las formas de desigual distribución de grupos de población en el territorio" (2002, p. 147). De ahí, que pueda ser pensada como una de las formas en que se expresa el proceso de diferenciación social o, lo que es igual, como la cristalización en el espacio de la estructura social (Machado Barbosa, 2001). Si relacionáramos esta idea al ámbito urbano, alcanzaríamos una definición como la de Sabatini, Cáceres y Cerdá (2001), para quienes la segregación residencial es "el grado de proximidad espacial o de aglomeración territorial de las familias pertenecientes a un mismo grupo social, sea que este se defina en términos étnicos, etarios, de preferencias religiosas o socioeconómicas, entre otras posibilidades" (p.27), para darle importancia al fragmento anteriormente descrito.

Para el trabajo dado hasta el momento podemos identificar que el estudio de fenómenos esta dado a partir de la forma como se definen y miden los conceptos fundamentales (Johnston et al., 2005: 1226). Como se mencionaba anteriormente la segregación sociao-espacial y residencial, debemos de entrelazar este fenómeno con el concepto central de la segregación como un ente solo, pero que esta articulado con la forma como se desea ser estudiado.

La segregación, tiene un sinnúmero de definiciones reportadas en la literatura, en esta sección se exploran algunos definiciones 
y apartados que nos aproximaran a poder entender su implicación con el campo de estudio. La definición clásica de segregación de Massey y Denton (1988) podría ser la más utilizada en la literatura latinoamericana. Es así, como la segregación es el grado en el que los individuos de diferentes grupos ocupan, viven, experimentan diferentes entornos urbanos en un espacio. Para ello se debe entender que la segregación está dada a medirse bajo indicadores dados en: i) Determine el entorno urbano (i.e. entorno social) de cada individuo, que se refiere a una enunciación de vecindad o contigüidad espacial (lo que en sí mismo resulta todo un problema metodológico, como se verá más adelante) y ii) Estime el grado en el que estos entornos o ambientes urbanos (i.e. entornos o ambientes sociales) difieren entre los individuos (Reardon y O'Sullivan, 2004).

De igual forma los diccionarios geográficos ofrecen definiciones de segregación que resultan similares. En estos términos, la segregación espacial es definida como la separación en el territorio de diferentes grupos de población. Así, un grupo está segregado espacialmente cuando sus miembros no se distribuyen en el territorio de manera uniforme respecto al resto de la población (Goodall, 1987; Gregory et al., 2009).

Según la literatura iberoamericana la tesis de Massey y Denton (1988) se ha consagrado como una de las definiciones más usadas, teniendo gran impacto. Para Castells (1974), la segregación socio-espacial en el ámbito urbano implica la distancia física entre la localización residencial de grupos sociales. Por tanto, la segregación es entendida como el fenómeno urbanístico socio-espacial en organizar de manera el espacio de manera homogénea. Generando ausencia o escasez relativa de mezclas socioeconómicas dentro de las unidades territoriales que integran la ciudad (i.e. por unidades territoriales o unidades espaciales se entiende: colonias, barrios, Áreas Geo-
Estadísticas Básicas, conocidas como ageb ${ }^{3}$ en México, entre otras). En esa medida se puede apreciar que la segregación socio-espacial, como el grado de proximidad espacial o la aglomeración geográfica de personas de una misma categoría social (Sabatini, 2000; Sabatini y Sierralta, 2006).

Retomando los apuntes de Sabatini, se aprecia que el concepto de segregación, segregación socio-espacial y residencial, está sujeto a factores de la aglomeración políticas públicas, políticas económicas y porque no como un resultado de la los procesos de la globalización. Es por ello que a la hora de estudiarla se debe rescatar la variedad de espacios urbanos (i.e. barrios, vecindarios, conjuntos de ageb), en los que los individuos del grupo residen mucho más cerca unos de otros de lo que lo se registraría en un patrón aleatoriamente distribuido (Allen y Turner, 2005).

Detallando los apuntes de Sabatini, se puede describir que la segregación residencial como socio-espacial, es diferente en la medida que se establecen criterios propios para su realización en el espacio.

\subsection{FUNDAMENTOS DE LA SEGREGACIÓN RESIDENCIAL EN LATINOAMÉRICA}

El proceso de la segregación residencial en las ciudades intermedias de América Latina, ha sido estudiado desde enfoques socioeconómicos, los cuales ha evidenciado que no son los únicos parámetros que existen para medir este fenómeno social que se da en el espacio, si bien para tener una visión más completa hay que tener en cuenta contexto de América Latina, como el migratorio, cultural, étnica y el

3 Según la definición de INEGI, una ageb urbana es un área geográfica ocupada por un conjunto de manzanas perfectamente delimitadas por calles, avenidas, andadores o cualquier otro rasgo de fácil identificación en el terreno y cuyo uso del suelo es principalmente habitacional, industrial, de servicios, comercial. 
desplazamiento por culpa del conflicto. Es así que se toma en consideración la historia del desarrollo urbano, cultural y socioeconómico de las ciudades, pues como bien argumentan Borsdorf (2003) y Janoschka (2002), la segregación residencial de toda ciudad se construye sobre la base de su estructura urbana heredada y sus transformaciones socioeconómicas. En lo que respecta a los estudios sobre este fenómeno en las ciudades Mexicanas, como Mérida. El proceso de la segregación residencial como socio espacial, ha tomado diferentes matices de desigualdad social, arrojando procesos estigmatizados en algunas partes de las ciudades. Dado a los procesos políticos e inmobiliarios que se están desarrollando en América Latina. De esta forma, existen otros fenómenos conductores que han influido y continúan influyendo en ella, como los propios procesos endógenos de agrupación de los grupos étnicos y la dinámica del mercado inmobiliario (Bayona i Carrarsco 2007, Sabatini 2003). En los últimos años se han desarrollado al menos tres cuadros interpretativos que buscaban explicar la segregación residencial en las ciudades occidentales, dados a: la ecología humana, el marxista y el económico. Pese a esto, todos los esquemas de estudio que se articulan en América Latina, frente a la segregación residencial, han sido criticados, lo que impide que sus argumentos sean "universalizados", en especial en el contexto de las sociedades tercermundistas contemporáneas (Estébanez 1992).

Al respecto, Sabatini (2003) deduce que procesos de la segregación residencial operan en las ciudades latinoamericanas, se pueden clasificar en dos tipos: a) Las motivaciones personales de los agentes, y b) Los factores sistémicos. Dada esta afirmación se deduce que existen unas premisas que permiten conceptualizar la segregación en América Latina.
En su primera apreciación, Sabatini (2003) incluye:

- Las ciudades de los países desarrollados, están dados a la construcción de una identidad pluriclasista, dada la que en las sociedades latinoamericanas se mezclan diferentes etnias, clases de estamentos que permiten su convivencia. De este modo se puede establecer que el modelo de dispersión del modelo de la ciudad favorece en cierta forma la disminución de la distancia y de igual manera la interacción entre grupos sociales.

- La obtención de bienes públicos como la seguridad ciudadana y el paisaje permiten ser alcanzados por algunos grupos, que tienen ciertos beneficios en poder adquirir estos bienes a comparación de otros.

- La utilidad de la propiedad del inmueble, dado a generar una rentabilidad, bajo los señalamientos de patrimonio o proyecto familiar. Es por ello que se preestablecen ciertos espacios con motivaciones de identidad social de grupos con altos y de bajos ingresos, permitiendo así la segregación voluntaria.

- La consolidación, afirmación y defensa de algunas identidades sociales, en las que pertenece las élites y los grupos emergentes.

Los factores sistémicos, para Sabatini (2003):

- Por la condiciones de las políticas neoliberales se consolidan los procesos de la liberalización de los mercados del suelo, la concentración por parte de algunos gremios inmobiliario con un capital exorbitante, las nuevas formas de vivienda, dadas a las nuevas tipologías de desarrollo urbano conocida como condominio o conjuntos cerrados. Se debe caracterizar que las nuevas disposiciones de residencia están dadas a hacer auto segregadas con el fin de mantener una tipología cerrada, es decir un poder simbólico mencionado por Pierre Bourdieu, donde señala 
que algunas sociedades o grupos interponen unas reglas sobre una población determinada, con el fin de mantener una homogeneidad en los comportamientos de sus habitantes, generando nuevas formas de segregación residencial en estas nuevas localizaciones.

- La propagación espacial de la especulación del suelo desde el centro y los barrios de alta renta al resto del espacio urbano, denominado así por Samuel Jaramillo, donde señala que el suelo tiene un valor y que este valor va a depender del lugar en donde se encuentre, beneficiando a unos y perjudicando a los que no tienen el ingreso suficiente para poder adquirirlo. Dado lo anterior no se debe dejar a un lado la dispersión de los desarrollos residenciales, comerciales y de servicios "modernos" que fomentan la especulación del suelo en todos los estratos socioeconómicos.

- La dispersión de muchos de los servicios e infraestructuras a cargo de los gobiernos (especialmente en materia de vialidades y transporte), lo cual comúnmente termina por beneficiar más a los promotores inmobiliarios y a los grupos socioeconómicos mejor posicionados dentro de la estratificación social existente.

La segregación de la vivienda formal de los hogares más pobres fuera de las ciudades como un todo es una tendencia contemporánea en América Latina. Dicho fenómeno ha llegado a ser el trazo predominante en ciudades como Bogotá, Santiago y Ciudad de México, entre otras. Resulta apresurado concluir que este tipo de segregación se debe al aumento de las desigualdades; se propone que tiene que ver con el funcionamiento de los mercados de suelo. En este contexto, ha sido determinante la convergencia de los siguientes hechos en el proceso de segregación residencial: predominio de la promoción inmobiliaria privada en el desarrollo urbano y persistencia de fuertes desigualdades sociales, que hacen crecer en forma significativa los precios del suelo. Esto, ligado a la mala distribución del ingreso, genera que los inmuebles queden por fuera del alcance de los individuos de bajos ingresos (Rubiano y Burbano, 2015).

\subsection{LUCES DE LA SEGREGACIÓN SOCIO ESPACIAL}

Para poder analizar los procesos mediante la cual se ha consolidado la segregación socio espacial, se debe tener en cuenta varios puntos de vista, las transformaciones tanto económicas como políticas, las nuevas tecnologías de la información y de la comunicación que se han presentado principalmente desde finales del siglo xx, han sido factores relevantes para poder comprender las trasformaciones del espacio, mediante la articulación de la financiarización y la mercantilización del mercado inmobiliario en la transformación y organización del territorio. En este sentido, el estudio de la segregación socio espacial desde la perspectiva urbana y como valor, se convierte en una vía posible para comprender las transformaciones que han producido tanto los agentes y actores sociales en la construcción del espacio urbano como resultado de la territorialización de sus acciones. (López, M. Alexandra, 2018).

Para poder comprender la segregación socio espacial, debemos de establecer procesos históricos y dinámicas de la estructura urbana, que nos permitan desenmarañar los patrones de segregación desde diferentes perspectivas; en la época colonial y del periodo de modernización, conocida en un primer momento como la ciudad compacta, comprendida entre el periodo de 1513 y 1820 y posteriormente hasta 1950, el modelo de diferenciación residencial tradicional se caracterizó por la localización de las actividades comerciales, administrativas y de uso residencial de la clase alta como los aristócratas; y clase media, como los comerciantes, mestizos, artesanos, maestros, 
entre otros. En el centro histórico, y cerca de él la ubicación de los grupos sociales de bajos ingresos en la periferia, es decir, la posición social en aquella época estaba relacionada por la distancia de acuerdo a la ubicación de la plaza central (Bäbr y Borsdorf, 2005). En esa época colonial (1513-1820) se puede decir entonces que la división social del espacio se evidenciaba de acuerdo a la casta, la raza y la riqueza de los individuos (Aprile y Mosquera, 1984, p. 85). Como muestra de esto, la densidad poblacional es mínima justo en donde se encuentran en su mayoría los equipamientos colectivos, caso contrario en donde la densidad poblacional máxima se encontraba más alejada de ellos.

Con el trasegar del tiempo, se inicia el desarrollo de las zonas industriales, dadas entre (1820-1950), las cuales se encontraban en inmediaciones de las vías ferroviarias o de comunicación, que para ese tiempo era el único transporte terrestre que minimizaba los costos. Se va cambiando del modelo concéntrico a uno orientado a un crecimiento lineal desde el centro, dadas a la dirección de las vías de comunicaciones, lo cual permitiría minimizar costos. En la primera mitad del siglo $x x$, este modelo se caracterizó por la presencia de espacios productivos manufactureros e industriales y áreas residenciales integradas por grupos sociales internamente homogéneos, por sus bajos ingresos cada vez más localizados en la periferia, mientras que la población de clase alta crecía en un sector exclusivo en forma de cono que tenía como vértice en el centro de la ciudad (Espinosa, 1992; Sabatini y Cáceres, 2005).

Según la anterior afirmación, se puede mencionar que a finales del siglo $x x$, el desarrollo económico estuvo enmarcado bajo los procesos de producción capitalista, cuya etapa se destacaba el neoliberalismo ${ }^{4}$, dando $4 \quad$ El neoliberalismo es la etapa de mercado más desarrollada del modo de producción capitalista, que se expresa además, en nuevas lógicas de planeación y ordenami- importancia a varios agentes que a juzgar por cada uno de ellos, jugaron un papel importante en movilizar flujos de dinero a cargo del mercado inmobiliario, agentes privados como el sistema bancario, los propietarios de la tierra y de los medios de producción en la mercantilización de la vivienda, los constructores y urbanizadores privados, reflejaron la transformación de los estilos de vida de los grupos poblacionales. De manera que la ciudad fragmentada de la época se transforma de una escala en la que se venía presentando la segregación socio espacial a una escala más pequeña, permitiendo identificar de manera más detallada el fenómeno de la segregación socio espacial a una segregación residencial. Visto de otra manera se posibilita el acceso a la vivienda de acuerdo al capital que poseen los grupos poblacionales objetivo, de modo que se produce una ciudad formal o planificada por la fuerzas del mercado y una ciudad informal o espontánea, propia de los espacios de reexistencia y reconstrucción de la vida de los desplazados por la violencia, la crisis rural, entre otros. (Rivera, 2013).

Dado lo anterior, se puede señalar que la producción social del territorio ha tenido transformaciones que se relacionan con los ciclos de la economía, donde la lógica de localización y el capital poseen actores sociales, que van a determinar el desarrollo de la producción social: "el capital, [...], aparece como organizador y estructurador territorial preponderante, basándose en los múltiples recursos y ventajas competitivas de cada porción del territorio metropolitano, extrayendo las plusvalías más cuantiosas precisamente de las singularidades y especificidades que hacen de cada fragmento el lugar óptimo para cada tipo de producción". (Ciccolella y Vecslir, 2010, p. 2)

$\overline{\text { ento territorial, que dispone de los recursos que están en el }}$ territorio para atender las diferentes lógicas y actividades del mercado independiente de quien se beneficie bajo fines específicos, por lo tanto no es un modelo que promueva la mitigación de la segregación en la ciudad (Torres et al., 2009). 
En este sentido, se modifica de alguna forma, el sentido de la segregación, donde está dada a la concentración del capital inmobiliario, cuyo énfasis esta dado a la promoción de la construcción de conjuntos cerrados, la innovación del producto, la estética, el embellecimiento, entre otros atributos que hacen que el espacio sea visto con atracción.

De esta manera desde el punto urbanístico, el cono como área concentrada de las élites pasa a estar dispersa por efectos de la concentración del capital y la liberalización de los mercados de suelo (Ríos, 2010; Sabatini y Cáceres, 2005).

Dado lo anterior, se puede apreciar que la posición social ya no determina la posición geográfica, es decir la distancia en el espacio, sino que se desarrollan varias lógicas y fronteras urbanas, en el que las ciudades cambian su forma de octagonal a tener un modelo fractal.
Debido a al consumo, es decir, que de acuerdo a su condición de adquisitiva va a depender su adquisición. De tal manera, la población se distribuye, en términos de gradiente, lo que sería "un fenómeno que toca a una gran parte del territorio y que acentúa las fronteras entre los diferentes barrios, incluso entre islotes, yendo al encuentro de la representación clásica de la pobreza" (Prévôt, 2000, p. 417). Los grupos poblacionales que se van segregando a través, de las "fronteras", son los que poseen el capital necesario y suficiente para crear espacios privilegiados, bien sea por razones económicas, políticas o culturales (Cortés, 2010; Hiernaux, 1999), desde ese punto de vista se presenta la precariedad de la condición humana, es decir, "son los portadores de las diferencias que nos provocan más miedos y contra los cuales nos trazamos las fronteras y levantamos los muros" (Cortés, 2010, p. 119).

Imagen 1. Patrón de segregación del periodo de modernización en Suramérica

\begin{tabular}{|l|l|}
\hline \multicolumn{1}{|c|}{$\begin{array}{c}\text { Patrón de segregación durante el periodo de } \\
\text { modernización }\end{array}$} \\
\hline $\begin{array}{l}\text { Se aprecia la configuración del espacio urbano altamente } \\
\text { diferenciado, con barrios exclusivos para las clases medio- } \\
\text { altas (cono o zona de privilegio -verde oscuro-), zonas } \\
\text { residenciales planeadas para la clase obrera (verde medio) y } \\
\text { áreas de autoconstrucción para la población no incorporada a } \\
\text { las actividades económicas formales (verde claro). }\end{array}$ \\
\hline
\end{tabular}

Fuente: Tomado de Sabatini y Cáceres (2005, p. 4).

Dicho de otro modo, el proceso de segregación no se da por razones ambivalentes, a través de dos formas, donde la globalización y transformación económica, hacen parte de este fenómeno. Por un lado, está la mala redistribución de los grupos poblacionales en el espacio urbano, generado a raíz de la incapacidad de las estructuras económicas para brindar los ingresos necesarios para que la población no se separe según características socioeconómicas, como el salario o la educación, que los hagan diferenciarse por grupos que internamente sean homogéneos pero entre ellos dispares. Por el otro, el reconocimiento fallido o desigualdad del estatus (Fraser, 2008), donde la población que son denominados desposeídos desde el ámbito social y económico son excluidos y relegados a las zonas que no solo con menores servicios y equipamientos sino que presentan una baja calidad en la adquisición de equipamientos, "así, se ha estigmatizado el rol de las expresiones culturales de quienes se dice, son indignos y no 
merecedores de habitar la ciudad. Al parecer, la ciudad moderna e industrializada facilitó todo este fenómeno" (Lacarrieu, 2007, p. 50).

\section{ASPECTOS SOCIALES DE SEGREGACIÓN SOCIO-ESPACIAL Y SEGREGACIÓN RESIDENCIAL}

Como se mencionó anteriormente, la diferenciación social, está relacionada con la diferenciación social entre la segregación y la residencial, se entrelazan en algunos sectores de la geografía, dado que la sociedad está condicionada a estar aislada de por la misma categoría social que está inmersa el grupo familiar, es por ello que la población tiende a agruparse o concentrarse en algún determinado lugar, que en este caso se cataloga como segregación por localización. Esta ópera cuando basados en la existencia de varios grupos sociales, no está dispersos dentro del territorio sino que se encuentran en una zona específica. Es decir corresponde a los "... elementos integradores de la sociedad, formas de asociación y organización de la sociedad civil, sus funciones y tendencias". (Montañez et al, 1994, p. 42).

De este modo nos conduce a que el territorio da a la población un proceso de identificación social, permitiendo generar diferentes tensiones relacionadas con la polarización social. En este sentido la segregación expresa en el espacio distintos tipos de desigualdades, constituye en él, una inmensidad de diferencias que se asientan, reproducen y se agravan en el espacio. En si la estructura espacial de la ciudad no solo refleja las asimetrías propias de la sociedad, sino también "retroalimenta una estructura social compleja en la que coexisten y se combinan procesos de diferenciación, desigualdad y exclusión" (Saraví, 2008, p. 27). Con esta hipótesis, se menciona que la segregación socio espacial tiene un lugar de causa y no como una mera consecuencia, defendida por dos perspectivas teóricas que, aunque diferentes, tienen más de un punto de contacto. El primero de ellos se refleja en varios autores como "efecto de barrio". Salvaguardando la premisa de que "el vecindario importa”, como lo señala Katzman (1999). La segunda perspectiva parte del supuesto, donde las comunidades con pocas oportunidades son aquellas que presentan inocultables déficits en rubros necesarios para que sus habitantes puedan desarrollar todas sus capacidades, permitiendo que exista la marginalidad de este grupo poblacional.

De esta forma se relaciona con la "geografía de las oportunidades" involucra, entre otros elementos, la escasez de establecimientos educativos, la falta de puestos de trabajo, la baja tasa de creación de empleo y una insuficiente capacidad para generar recursos fiscales (Cáceres y Sabatini, 2004; y Galster y Killen, 1995).

Es así, como se inicia a estructurar la sociedad, generando la manifestación de colonias o sectores cerrados con ciertas peculiaridades, que van a distinguirse de los otros, sea por tener altos ingresos o por el contrario bajos ingresos.

\subsection{Personalización Espacial y Territorial}

Dentro de los estudios dados en la personalización espacial y territorial, se parte de la premisa de poder identificar el concepto de comunidad, cuyo significado esta dado a la interacción, comportamientos de las personas que habitan en un espacio determinado, cuya unión está dada en los vínculos que se dan dentro de sus habitantes. Así por ejemplo, se verifica una fuerte segregación residencial en una sociedad caracterizada por su proximidad y la integración social entre las diferentes clases sociales, lo cual viene acentuándose durante los últimos quince años (Katzman y Retamoso 2005, Observatorio Social IMM 2007, 
Veiga y Rivoir 2008). En las áreas urbanas, la comunidad se establece como aquella uniones que se dan entre vecinos del mismo barrio, así como cualquier otra división que se de en el territorio, permitiendo la unión de lazos sociales, económicos, políticos y culturales que se van reforzando con la interacción entre sus miembros, por lo que permite la transformación del espacio en un lugar a su imagen y semejanza. Es así, como Rossi (1999), menciona que los grupos sociales van determinando las condiciones de su espacio, de ahí la importancia de la manipulación y transformación de su espacio y territorio, y que va a depender el desarrollo de su identificación social y cultural, pero hay momentos en los cuales este proceso no resulta ser favorable para el grupo social, en ese sentido se pliega a ciertas condiciones, como es el caso de las tensiones dentro de los mismos habitantes por defender su espacio y lo que realmente lo ha identificado.

Danilo Veiga (2003), explica la importancia de la relación de espacio y la conducta humana como parte de un proceso de identificación territorial, basada en las características culturales y los modos de vida de los habitantes.

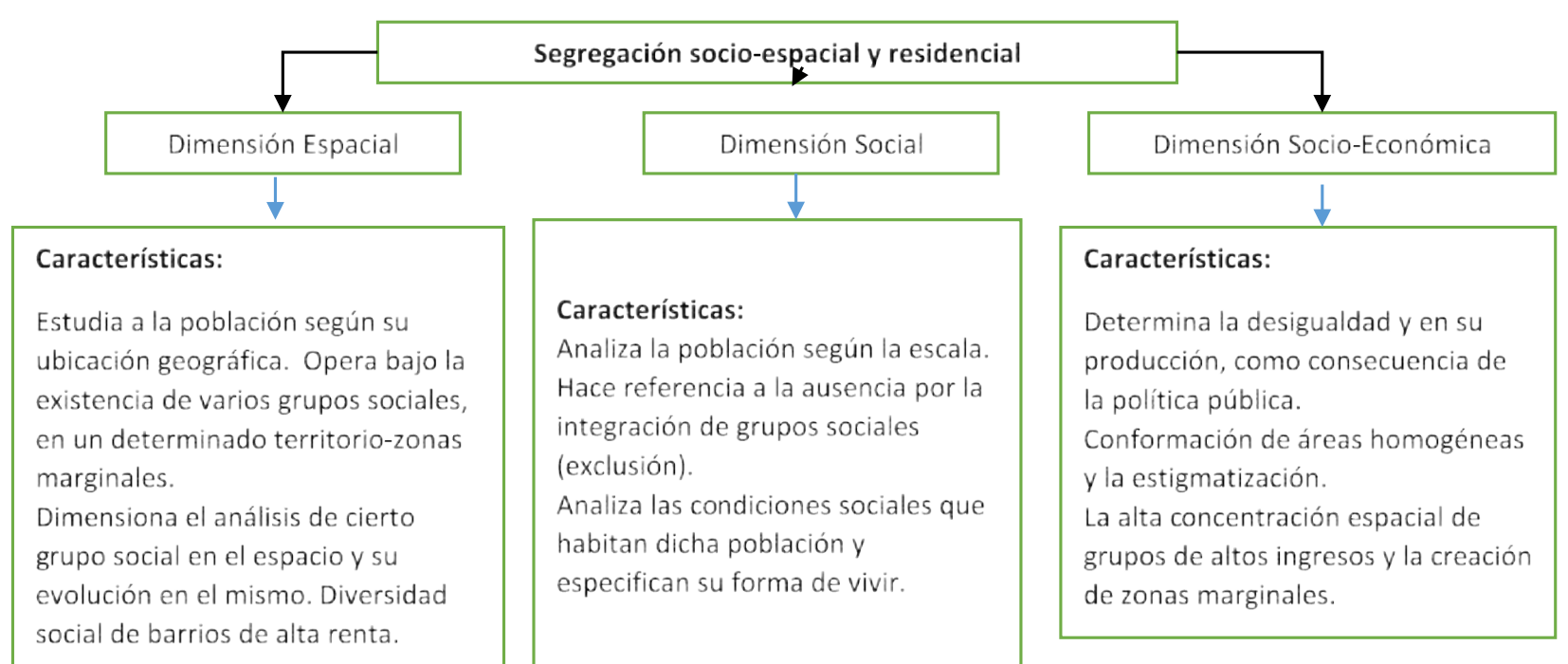

Imagen 1. Esquema teórico propuesto para entender las dimensiones de Segregación Socio-espacial y Residencial Fuente: Elaboración propia (2018)

\section{METODOLOGÍA}

El método utilizado en este estudio es descriptivo, a partir del objetivo de aproximarse conceptualmente y metodológicamente a los procesos como se han sabido llevar el fenómeno de la segregación socio espacial y residencial para el caso de Latinoamérica, desde la articulación entre los conceptos de segregación socio espacial y residencial. El diseño del estudio se basa en dos fases: i) etapas de revisión bibliográfica, informes, trabaos de grado, sobre la manera de como se ha estudiado la segregación socio-espacial y residencial y ii) análisis de su aplicabilidad. Se basa en dos categorías centrales: territorio y espacio, con una variables ordenamiento territorial.

\section{REFLEXIONES FINALES}

Después de este amplio recorrido conceptual y metodológico dado a contextualizar la segregación socio espacial y la residencial, se destaca que el primer término es de carácter 
espacial, donde se señala una nueva forma de segregación residencial en la naciente ciudad postmoderna: un modelo que deja de ser a gran escala, como ocurría en la ciudad industrial, para ser ahora de pequeña escala; una situación donde distintas razas y clases sociales residen de manera próxima en el espacio, pero que gracias a distintos mecanismos, como cámaras de video y sistemas de vigilancia proporcionados por compañías privadas de seguridad, se encuentran efectivamente separados (Davis 1990).

El epítome de este modelo de segregación se encuentra en la aparición masiva de condominios cerrados (gated communities): grandes y medianos complejos residenciales, ubicados tanto en la periferia urbana como en zonas antiguamente pobres, normalmente cercados por muros, barricadas $\mathrm{u}$ otras formas de separación física que permite su aislamiento, a pesar de la cercanía en el espacio, de los sectores empobrecidos que viven alrededor (Davis 1990, Caldeim 2000). De esta forma la ciudad abandona su antigua geografía sectorial para convertirse en un collage sin patrón alguno de islotes de pobreza y riqueza, próximos unos de otros, pero separados por estas nuevas tecnologías militarizadas.

De este modo se podría precisar que de nada sirve tener buenos documentos técnicos que no puedan ser aplicados, ni generar leyes que no se cumplen, ni seguir formulando políticas desde el escritorio que resultan en algunos casos ineficientes, con el supuesto de mejorar las condiciones de vida de la población, cuando no es así.

Las esperanzas si se puede llamar así, están dadas en el ordenamiento territorial, dado a que allí se plasman ideas, directrices, proyectos que permite darle visión al territorio y con ello beneficiar a la población, como es de saberse los impactos de cualquier plan no han alcanzado las expectativas, como consecuencia de la concentración del poder político, económico, social, ambiental y territorial. Dado a esto nos lleva a la intriga de poder identificar del porque suceden fenómenos que en ocasiones no son evitados, si no por el contrario, son llevados de manera aislada, perjudicando las formas de igualdad y equidad que se plasmadas en el territorio.

Con el proceso urbanístico algunos autores han notado el surgimiento de un patrón urbano similar en algunas ciudades de América Latina y con ello la aparición de estos condominios para clases altas en las zonas tradicionalmente empobrecidas de la ciudad. Los casos más dados este tipo de análisis están dados a ciudades como Sáo Paulo (Caldeira 2000) y Santiago de Chile (Cáceres y Sabatini 2004), Buenos Aires, Montevideo y Río de Janeiro (Portes et al. 2005), dando origen a lo que se ha denominado la ciudad fragmentada (Bahr y Borsdorf 2005) o segregación de pequeña escala (Sabatini 2003), dada a la proliferación de pequeños enclaves autosuficientes de riqueza, en medio de un continuo de pobreza urbana.

El análisis crítico e interpretativo sobre el fenómeno de la segregación, ha existido siempre, porque la desigualdad es persistente, continua, más aun, debemos diferenciar ambos conceptos, la desigualdad es persistente, la segregación no, debido a su capacidad de dinamismo y transformación, donde se señalaba anteriormente.

La segregación residencial es estudiada desde una aproximación cuantitativa, como cualitativa. Donde las alternativas cuantitativas, son utilizadas en la construcción y mapeo de cocientes de localización y el cálculo de índices de segregación. Por otro lado las alternativas cuantitativas tienen procedimientos estadísticos, donde son elaborados desde el punto de vista de la ecología factorial. Por su 
parte, la aproximación cualitativa al estudio de este fenómeno se enfoca en el análisis de los procesos de desarrollo urbano y socioeconómico que se dan en las ciudades.

La noción segregación residencial posee un fuerte e intrínseco carácter espacial, su aplicación suele suscitar infinidades discrepancias acerca de su conceptualización y aproximación metodológica. En este trabajo la segregación residencial se conceptualiza como la distribución espacial de los diferentes grupos sociales que integran una población urbana y que tienden a conformar áreas espaciales socialmente homogéneas a partir de la acción de los mecanismos de diferenciación social, las decisiones y motivaciones individuales y un conjunto de factores sistémicos desde un aspecto micro espacial. $\mathrm{Y}$ por el contrario la segregación socio-espacial trabaja aspectos globales, donde no se logra diferenciar los aspectos económicos, sociales desde la vivienda o el lugar donde reside un grupo familiar.

Desde un aspecto general se puede mencionar que el fenómeno de la segregación en una oportunidad para el reforzamiento de las identidades quebrantadas producto de la alienación de la sociedad. Es importante señalar que se debe mirar con una capacidad crítica capaz de formular respuestas y alternativas, aun en el escenario más oscuro, solo convirtiendo el problema en oportunidad para poder identificar la dimensión que se pierde cuando se elaboran las políticas por parte del Estado.

\section{REFERENCIAS BIBLIOGRÁFICAS}

Aguilar, Adrián y Pablo Mateos. 2011. "Diferenciación sociodemográfica del espacio urbano de la Ciudad de México". EURE 37 (110): 5-30.
Amendola, G. (2000). La Ciudad Postmoderna. Magia y Miedo de la Metrópolis Contemporánea. Madrid: Celeste.

Aprile, J. y Mosquera, G. (1984). Clases, Segregación y Barrios. Cali, Colombia: Universidad del Valle.

Arriagada, C. y Rodríguez, J. (2003). Segregación residencial en áreas metropolitanas de América Latina: magnitud, características, evolución e implicaciones de política. Santiago de Chile: CEPAL.

Arriagada, C., \& Rodriguez, J. (2004). "Segregación residencial en la ciudad latinoamericana". EURE (Vol. XXIX, $N^{\circ} 89$ ), Santiago de Chile, 5-24.

Bäbr, J. y Borsdorf, A. (2005). La Ciudad Latinoamericana: La construcción de un modelo. Vigencia y perspectivas. Revista de Ciudad, Urbanismo y Paisaje Ur[b] Es, 207-221.

Bäbr Jürgen \& Borsdorf Axel (2005), La ciudad Latinoamericana. La construcción de un modelo. Vigencia y perspectivas. Perú. Hozlo s,r,l. pp. 208-2012.

Bayona i Carrarsco, Jordi. 2007. "La segregación residencial de la población extranjera en Barcelona: ¿una segregación fragmentada?". Scripta Nova XI (235)

Borsdorf, A. Cómo modelar el desarrollo y la dinámica de la ciudad latinoamericana. EURE (Santiago). [online]. 2003, vol.29, no.86 [cited 10 Feb 2008], p.37-49. Available:

Borsdorf, Axel. 2003. "Cómo modelar el desarrollo y la dinámica de la ciudad latinoamericana".

EURE XXIX (86): 37-49.

Briggs, Xavier; Darden,Joe, \& Aidala, Angela (1999). In the wake of desegregation: 
Early Impacts of scattered-site housing on neighborhoods in yorkers. New York. Journal of the American Planning Association, 65, 27-68

Buzai, Gustavo (2003). Mapas sociales urbanos. Buenos Aires, Lugar Editorial.

Brun, J. (1994). Essai critique sur la notion de ségrégation et sur son usage en géographieurbaine. En Brun, J. y Rhein, C. (Eds.), La Segregation dans la ville (pp. 21-58). Paris: L'Harmattan.

Cabrales B. L. (2003). Estado del conocimiento sobre las urbanizaciones cerradas en Iberoamérica. Unesco. $\mathrm{N}^{\circ}$ (59). 58-64.

Cáceres, G., \& Sabatini, F. (2004). Barrios cerrados en Santiago de Chile: entre la exclusión y la integración. Santiago: Lincoln Institute of Land Policy / Pontificia Universidad Católica de Chile.

Carrasco Bayona, Jordi (2007). La segregación residencial de la población extranjera en Barcelona: ¿una segregación fragmentada? Revista Electrónica de Geografía y Ciencias Sociales, XI (235).

Castells, Manuel. 1986. La cuestión urbana. México: Siglo XXI.

CEPAL. (2010). "El lugar importa: disparidades y convergencias territoriales". En CEPAL, "La hora de la igualdad. Brechas por cerrar, caminos por abrir: trigésimo tercer periodo de sesiones, Brasilia, 30 de mayo a 1 de junio 2010" (págs. 131-157). Santiago, Chile: CEPAL.

Ciccolella, P. y Vecslir, L. (2010). Nuevos espacios del terciario y transformación metropolitana en Buenos Aires. XI Seminario Internacional RII y IV Taller de Editores RIER Universidad Nacional de Cuyo. Universidad Nacional de Cuyo.
Esteban, Alfonso, Curiel, Javier \& Perelló, Salvador(2003). Inmigración y segregación urbana. Papeles de Economía Española. 98, 262-273

Espinosa, M. A. (1992). Crecimiento Urbano de Ibagué 1938-1989. Universidad Pedagógica y Tecnológica de Colombia.

Fernández, R. (1996). La Explosión del Desorden. La Metrópoli como Espacio de la Crisis Global. Madrid: Editorial Fundamentos.

FORD, Larry (1996), "A new and improved model of the Latin American city structure", Geographical Review, 86, 3, pp. 437---440.

Chávez Reyes, Héctor Saúl y Fitch Osuna, Jesús Manuel (2009), "La composición de fragmentos urbanos y su efecto en el mercado inmobiliario" en Revista ASINEA, Octubre 2009, Numero 35, ASINEA, México.

Checa, J. C., \& Arjona, Á. (2007). "Factores explicativos de la segregación residencial de los inmigrantes en Almería". Revista Internacional de Sociología, 173-200.

Cortés, J. M. (2010). La ciudad cautiva. Control y vigilancia en el espacio urbano. Madrid, Espña: Akal S.A.

Fraser, N. (2008). Escalas de Justicia. Barcelona, España: Herder Editorial, S. L., Barcelona.

Garín, A., Salvo, S., \& Bravo, G. (2009). "Tendencias en la segregación residencial en Chile". Revista de ciencias sociales FACESLUZ, 407-418.

Galster, G., \& Killen, S. (1995). The Geography of Metropolitan Opportunity: A Reconnaissance and Conceptual Framework. Housing Policy Debate, 6(1), 7-43. 
Harvey, David (1992), Urbanismo y desigualdad social, Siglo XXI, México.

Harvey, D. y Smith, N. (2005). Capital financiero, propiedad inmobiliaria y cultura. Barcelona, España: Universitat Autonoma de Barcelona.

Harvey, D. (2013). Ciudades rebeldes: Del derecho de la ciudad a la revolución urbana. Madrid: Akal S.A.

Hidalgo, R. (2004). De los pequeños condominios a la ciudad vallada: las urbanizaciones cerradas y la nueva geografía social en Santiago de Chile (1990-2000). EURE, 30(91), 29-52.

Hiernaux, D. (1999). Los frutos amargos de la globalización: expansión y reestructuración metropolitana de la ciudad de México. EURE (Santiago), 25 (76), 1-14.

Janoschka, M. (2002). "Urbanizaciones privadas en Buenos Aires: ¿hacia un nuevo modelo de ciudad latinoamericana?", Cabrales, L. F. (ed.), Latinoamérica: Países abiertos, ciudades cerradas. Guadalajara: Universidad de Guadalajara/ UNESCO, 287-318-

Janoshcka, M. y Glasze, G. (2003). Urbanizaciones cerradas: un modelo analítico. CIUDADES. Puebla.

Katzman, R. y Retamoso, A. (2005) "Segregación espacial, empleo y pobreza en Montevideo". Rev. CEPAL, No. 85. Santiago. Koch, M. y Valdés, E. (2008) Una mirada

Keil, R. (2018). Suburban Planet. Polity Press.

Lacarrieu, M. (2007). La "insoportable levedad" de lo urbano. Eure, 33(99), 47-64. En línea.

Lévy, J. P., \& Brun, J. (2002). De la extensión a la renovación metropolitana: mosaico social y movilidad. En Dureau, F. et al. (Comp.), Metrópolis en movimiento: Una comparación internacional (pp. 147-165). Bogotá: Instituto de Investigaciones para el Desarrollo.

López, M. Alexandra (2018) Segregación socio espacial bajo el nuevo modelo de ciudad en América Latina Características, perspectivas e implicaciones. Hallazgos, 15(30), 99-124.

Machado Barbosa, E. (2001). Urban Spatial segregation: foundation for a typological analysis. International Seminar on segregation in the city. Cambridge: Lincoln Institute of Land Policy.

MASSEY, D. S. \& Denton, N. A. (1988). "The Dimensions of Residential Segregation". Revista Social For-ces, vol. $67, \mathrm{~N}^{\circ} 2$. The University of North Carolina Press

Moura, R. (2003). Inversiones urbanas en el contexto de la competitividad y globalización: los eventos en Curitiba. EURE (Santiago), 29 (86), 51-68.

Mnsstu, Douglas y Nancy DENTON 1988 Jhe Dimensions of Residential Segregationa. Social Forces, vol. 67, N" 2 ..

Ortiz, J. \& Schiappacasse, P. (1998). Dimensiones latentes de la diferenciación del espacio social en una metrópolis latinoamericana: El caso del Gran Santiago. Geographicalia, 36, 111 - 130

Palacios, A. (2015). Impacto socio espacial de la implantación de centros comerciales en Bogotá D.C. Perspectiva Geográfica, 20(2), 319-338.

Prévôt, M.-F. (2000). Segregación, fragmentación, secesión. Hacia una nueva geografía social en la aglomeración de Buenos Aires. Economía, Sociedad y Territorio, II (7), 405-431.

Prévot-Shapira (2001), "Fragmentación espacial y social: conceptos y realidades" en Perfiles latinoamericanos, № 19, FLACSO, México. 
Ríos, M. (2010). Segregación Residencial; el Problema Social Desde la Perspectiva Económica y Urbana. Universidad Nacional de Colombia. Bogotá, Colombia.

Rivera, J. A. (2013). Proceso de urbanización y agentes urbanos Directores: Horacio Capel Sáez Territorial y Gestión Ambiental Universidad de Barcelona Facultat de Geografia i Historia. Universidad de Barcelona.

Roca, Miguel (2012). Segregación social: distribución y construcción espacial en Lima Metropolitana. Revista Espacio y Desarrollo $N^{\circ}$ 24, 119-134.

Rossi, Aldo (1999), La ciudad de la Arquitectura, Gustavo Gili, Barcelona.

Salinas Varela, E. (2009). Fragmentación urbana y su relevancia en la planificación urbana y territorial actual. Ignire - Centro de Estudios de Política Pública.

Sabatini, F., Cáceres, G., \& Cerdá, J. (2001). Segregación residencial en las principales ciudades chilenas: Tendencias de las tres últimas décadas y posibles cursos de acción. Revista eure, 27(82), 21-42.

Sabatini, F. y Cáceres, G. (2003). Relación entre promoción inmobiliaria y la segregación residencial. Giros insospechados de la ciudad latinoamericana. Santiago de Chile.

Sabatini, F. \& Cáceres, G. (2004). Los barrios cerrados y la ruptura del patrón tradicional de segregación en las ciudades latinoamericanas: el caso de Santiago de Chile. En G. Cáceres \& F. Sabatini (Eds.), Los barrios cerrados en Santiago de Chile: entre la exclusión y la integración social(pp. 9-43). Santiago: Lincoln Institute of Land Policy.
Sabatini, F. y Cáceres, G. (2005). Relación entre Promoción Inmobiliaria y Segregación Residencial: Giros Insospechados de la Ciudad Latinoaméricana. Lincoln Institute of Land Policy. En línea. Recuperado de: https://doi. org/10.1017/ CBO9781107415324.004 